\title{
Knowledge-Based Approaches for Scheduling Problems: A Survey
}

\author{
S. J. Noronha and V.V.S. Sarma, Senior Member, IEEE
}

\begin{abstract}
Scheduling is the process of devising or designing a procedure for a particular objective, specifying the sequence or time for each item in the procedure. Typical scheduling problems are railway time-tabling, project scheduling, production scheduling, and scheduling computer systems as in flexible manufacturing systems and multiprocessor scheduling. Further, there are a number of related problems belonging to the larger class of planning problems, such as the early stage of project management and resource allocation in a job shop.

Scheduling is a rich area demanding the application of efficient methods to tackle the combinatorial explosion that results in real world applications. Recent developments in artificial intelligence (AI) have led to the use of knowledge-based techniques for solving scheduling problems. In this paper, we survey several existing intelligent planning and scheduling systems with the aim of providing a guide to the main AI techniques used. In view of the prevailing difference in usage of the terms planning and scheduling between AI and OR, we present a taxonomy of planning and scheduling problems. We illustrate the modeling of real world problems from closed deterministic worlds to complex real worlds with the project scheduling example. We survey some of the more successful planning and scheduling systems, and highlight their features. Finally, we consolidate the AI approaches to knowledge representation and problem solving in the project management context.
\end{abstract}

Index Terms- Intelligent systems, knowledge-based systems, planning, project management, scheduling.

\section{INTRODUCTION}

$\mathbf{S}$ CHEDULING is the process of devising or designing procedure for a particular objective, specifying the sequence or time for each item in the procedure. Typical scheduling problems are railway time-tabling, project scheduling, production scheduling, mass transit scheduling, hydropower scheduling, scheduling nurse shifts in a hospital, etc. Emerging application examples of scheduling in computer systems are in flexible manufacturing systems, multiprocessor scheduling, robot activity scheduling, scheduling in very large scale networks and hard real-time scheduling. Further, there are a number of related problems belonging to the larger class of planning problems, e.g., the early stage of project management, and resource allocation in a job shop. Even this small sample shows the wide range and diversity of scheduling problems, and their importance in almost every sphere of human life stems from the need to utilize limited resources in as efficient a way as possible, simultaneously satisfying several domain-specific constraints.

Manuscript received October 1, 1989; revised October 1, 1990.

The authors are with the Department of Computer Science and Automation, Indian Institute of Science, Bangalore 560 012, India.

IEEE Log Number 9144303.
Classical approaches to scheduling problems, as practiced in the management science and operations research (OR) fields, are characterized by a reduction of the problem into a mathematical programming formulation, and subsequent solution by formal algorithmic methods. These approaches face the difficulty that the problem quickly becomes computationally infeasible for even moderately big problems, due to the large number of variables and inherently large search space generated by the model. An additional disadvantage is that such models do not directly reflect the natural structure of the domain, and hence make it difficult to employ domainspecific knowledge to reduce search. In recent years, some OR researchers have been advocating the use of simple heuristic rules to tackle large problems, but these lack the advantage of a unified approach and are seen as a poor relation to the formal methods.

Increasing awareness of recent developments in artificial intelligence (AI) is paving the way for the widespread use of knowledge-based techniques for solving the classical scheduling problems [24], [27]. AI provides a large body of tools and techniques such as informed search methods, sophisticated knowledge representation schemes, and high-level programming environments. Many of these methods have already been applied to real world scheduling problems, and systems have been built that continue in good use today. In this paper, we survey several existing intelligent planning and scheduling systems with the aim of providing a guide to the main AI techniques used, and focus on project scheduling as an example problem rich enough to demonstrate the use of these techniques.

In view of the prevailing difference in usage of the terms planning and scheduling between $\mathrm{AI}$ and $\mathrm{OR}$, and in order to bring the relations between various problems into perspective, we present a taxonomy of planning and scheduling problems in Section II. We illustrate the modeling of realworld problems from closed deterministic worlds to complex real worlds with the blocks world and project scheduling examples. In Section III, we survey some of the more successful planning and scheduling systems, and highlight their features. Section IV attempts to consolidate the AI approaches to knowledge representation and problem solving in the project management context.

\section{Planning and Scheduling Problems: A Taxonomy.}

The term scheduling differs in usage between different fields of research. In OR a distinction is made between sequencing and scheduling: a sequence is simply the order in which 
activities (jobs) are processed (on some machines), whereas a schedule contains explicit information about the start and finish times of the various operations [20]. Time-tabling is the process of deriving a schedule from a sequence. A distinction is also drawn between planning and scheduling: in project management, the former refers to the initial stage of a project during which activities are defined and their durations, precedence relationships, and other attributes are specified; whereas scheduling is the second stage during which the activities to be done are selected, sequenced, and time-tabled in a way that satisfies resource and timing constraints and meets certain objectives such as minimizing project costs [67]. However, the term planning is also used for other problems such as facilities' location planning and water resources planning, which are not scheduling problems. In comparison, in AI, the terms planning and scheduling are used interchangeably and include plan generation, selection, and coordination [11]. Additional confusion is caused by including allocation of resources as part of the scheduling definition (e.g., [21]); however, problems such as the classical PERT/CPM project scheduling problem do not consider resources. This variance in usage requires that we begin with a definition of the terms, and this section therefore presents a taxonomy of planning and scheduling problems. Our definitions strive to be as general as possible while providing a useful characterization of the different problem classes.

We define planning as the process of devising, designing, or formulating something to be done, such as the arrangement of the parts of a thing or an action or proceeding to be carried out. We call the latter form of planning activity planning. Activity planning corresponds to the use of the word planning in the AI literature and includes problems such as robot activity planning and project planning. Planning problems that do not fall under this category are facilities' location planning, vehicle routing problems, and resource allocation problems such as the effective allocation of a set of weapons to a set of targets [71], and the classical transportation problem [31].

Scheduling is the process of devising or designing a procedure for a particular objective, specifying the sequence and/or time for each item in the procedure. By this definition, scheduling is a subset of activity planning, and hence, of planning. The identifying characteristic of a scheduling problem is the requirement of specifying timing or sequencing information for each item (activity) in the procedure. Further, in contrast to the definitions of planning and activity planning, the word "formulating" is conspicuous by its absence: in keeping with tradition we have restricted the scope of scheduling problems to preclude the early planning stage in which the activities and their attributes are defined.

Many planning and scheduling problems also come under the category of design problems. Following Goel and Pirolli [25] we define design as the process that takes as input a set of goals and intentions, and outputs a specification of an artifact, with the process being characterized by 1) a usually incomplete problem description, 2) limited or delayed feedback from the world, 3) costs associated with every action in the world, and 4) the artifact functions independently of the designer. Examples of design problems are: doing a VLSI

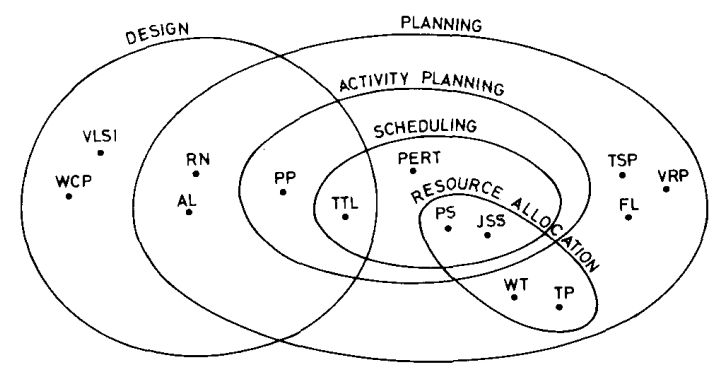

Fig. 1. A taxonomy of planning and scheduling problems.

$$
\begin{array}{ll}
\text { AL } & \text { - planning an automobile assembiy line } \\
\text { FL } & \text { - facilities location problem } \\
\text { JSS } & \text { - job-shop scheduling } \\
\text { PP } & \text { - project planning } \\
\text { PERT } & \text { - uniimited-resource project scheduling } \\
\text { PS } & \text { - project scheduling } \\
\text { RN } & \text { - designing a new railway network } \\
\text { TP } & \text { - classical transportation problem } \\
\text { TSP } & \text { - travelling salesman problem } \\
\text { TTL } & \text { - time-tabling of lectures } \\
\text { VRF } & \text { - vehicle routing problem } \\
\text { VLSI } & - \text { doing a VLSI layout } \\
\text { WCP } & \text { - writing a computer program } \\
\text { WT } & \text { - allocation of weapons to targets }
\end{array}
$$

layout, writing a computer program, and designing hard realtime systems [74]. We provide this definition for completeness, and we shall not further discuss design problems and strategies in this paper.

The classification we have proposed may be visualized with the aid of the Venn diagram in Fig. 1. Typical examples are marked as points in the appropriate sets. However, before we proceed, we make two important observations. First, the classification sets are not crisp. That is, a given problem may belong to more than one category, with varying degrees of membership, and the extent to which it is a "planning" or "design" problem depends on how much it deviates from the definitions and prototypical problems of these categories.

Second, we have attempted in our definitions to capture the essence of each problem category. However, each type of scheduling problem can and should be characterized along several other important dimensions: determinism versus stochasticity (fixed task durations, or probabilistic durations); complexity (polynomial versus NP-hard); real-time requirements (hard real-time constraints versus off-line solution); the presence or absence of precedence and timing constraints, e.g., task coupling or deadlines on activity processing; presence of resource constraints; the scheduling objectives (minimizing makespan, cost, utilization, etc.); optimizing versus satisficing requirements of the problem; sequencing versus time-tabling; etc. 


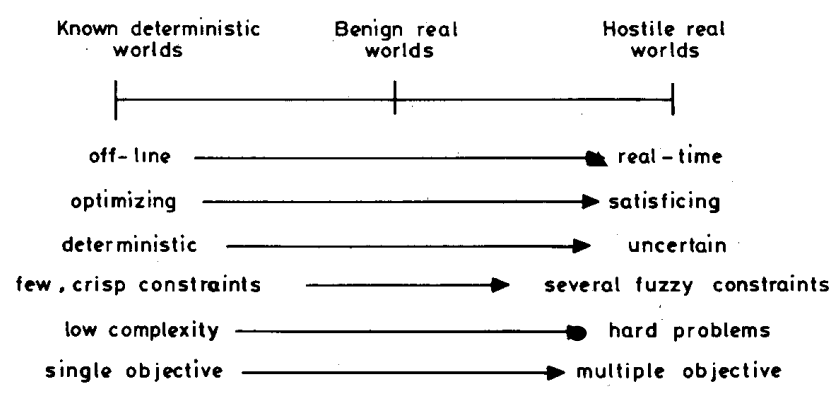

Fig. 2. The spectrum of scheduling models.

All these criteria may be used to subclassify scheduling problems, but it is more instructive and fruitful to identify the unifying thread that runs through all of them: the gradual progression from simplified, abstract, idealized formulations to real-world complexity. For convenience we classify planning and scheduling problems into three broad categories along this dimension (Fig. 2):

- Problems in known deterministic worlds.

- Problems in benign real worlds.

- Problems in hostile real worlds.

The first category is typified by the well-known missionaries and cannibals problem in which three cannibals and three edible missionaries seek to cross a river [3]. A single boat is available which can carry at most two people, but if the missionaries should ever be outnumbered by the cannibals at any time, dire consequences will result! The problem is to find a safe schedule of crossings for the entire group. Implicit in this problem is the closed-world assumption: we do not consider whether there is a bridge nearby, or whether anybody can swim! Problem-solving of this kind would not provide the best solution in the real world, but because of the simplicity of such problems, most of the traditional formulations of planning problems assume a known deterministic world.

The second category recognizes the need for explicit techniques for handling uncertainty and imprecision in the problem domain, e.g., in activity durations and resource estimates. The "optimal" shortest-duration schedule may not be useful in a real-life scheduling problem, since it is probable that some activities will be delayed or postponed. What is required is a "robust schedule" that is less sensitive to delays, rather than an optimal one. It is evident that heuristic knowledge has a prominent role to play in such planning and scheduling problems. A discussion of reactive and real-time planning and related concepts appears in Section III-E.

Scheduling activities in times of tension, war, and political unrest fall into the third category, viz., scheduling in hostile real worlds. In these situations, the consequences of not meeting deadlines may be serious. Scheduling algorithms have to distinguish between critical deadlines and soft (flexible) constraints, and adjust the plans accordingly. The premium on time may mean that an inexact but fast answer would be preferable to an accurate but slow answer. In many real-time systems there is no incentive to perform optimization, apart from meeting deadlines [74].
Any given problem can be modeled with a varying degree of correspondence to the real world, and the usefulness of a model is a function of both its ease of solution and its accuracy. However, these two constitute a tradeoff. Because of the richness of real-world problems, and the resulting variation in model formulation, it is more appropriate to refer to jobshop scheduling and project scheduling as classes of problems rather than individual well-defined problems (i.e., as sets rather than points in Fig. 1). In the following sections we illustrate the above classification with two examples: planning in the blocks world and project scheduling.

\section{A. The Blocks World Class of Problems}

The blocks world has been a popular test domain in AI for various planning systems. It consists of a robot in a world of blocks of various sizes which are numbered with the letters of the alphabet. The robot can perform elementary actions such as picking up a block, putting it down on the table or on another block, etc. The planning task is to devise a sequence of such primitive actions that results in a given "goal configuration" of the blocks, e.g., to create a single stack of blocks in alphabetical order. This domain is clearly a closed and deterministic world and is positioned at the leftmost end of our spectrum.

This simple blocks world problem can be extended by not specifying the initial configuration. The problem is now to find a plan for transforming any given configuration into the goal stack. Since the plan must work independently of the state of the world, the plan is not just a single sequence of actions but a prescription of the action(s) to be taken in each situation in the external world, i.e., a strategy. This type of planning, and planning in uncertain domains as discussed below (reactive planning) are often studied under the label of "situated action" [68], [69].

The initial state-less blocks world problem is brought still closer to the real world by introducing into it a playful baby (Schoppers' [68]) that wanders around the room while the robot is at work, occasionally knocking blocks down and thus introducing an element of uncertainty into the domain. In this benign real world too, the nature of the plan is a strategy: what should be done when each unexpected event occurs. The strategy may be viewed as a tree (more generally an acyclic directed graph) with each node representing an external event or situation (and the associated decision question of action 
selection), and each arc representing the selected action. The leaf nodes of the strategy are either successful situations (states in which the goal has been achieved) or failure situations in which it has become impossible to achieve the goal. We note that despite the presence of uncertainty this version should be considered a "closed" world because all the various types of events that could occur are known in advance, even though we do not know when these events might occur. There is a quantum jump in complexity between this closed dynamic world and the following version of the blocks world problem which is a truly "open" world.

Schoppers' baby is sometimes mischievous. In its more recalcitrant moods it actually tries to undo the robot's efforts snatching away blocks from his hands or even throwing blocks at the robot. In this "hostile" real world a robot planner may actually have to play a game with the baby, hiding blocks, distracting the baby's attention by building a dummy stack at another corner of the room, or locking up the baby in the bathroom. This category of problems is the hardest indeed because of the openness of the world and the consequent wealth of "consensus reality" [41] or commonsense knowledge required by the planner, and no AI planner has attempted this type of planning yet.

\section{B. The Project Scheduling Class of Problems: Definitions and Classical Approaches}

A project is a set of interrelated activities which utilize various resources and possess the following characteristics [48]: 1) A project is finite, and has a definite goal. 2) It is homogeneous, i.e., the individual activities of the project can definitely be identified as belonging to it. 3 ) It is complex, involving a number of parallel activities with a significant interplay of skill, materials, and facilities. 4) It is usually nonrepetitive. Each activity has a particular duration, and uses a specified amount of resources. There may be precedence constraints on the order in which activities can be executed timing constraints such as release times and deadlines to meet, and constraints on the availability of resources. The problem is to find a schedule, i.e., an assignment of a start time and resources to each activity, such that some scheduling objective such as minimizing overall project duration is achieved.

Early work on the project scheduling problem, i.e., PERT and CPM [43], [79] dealt with a simplified version of the problem and modeled it in a known deterministic world: activity durations were assumed fixed, and resource and timing constraints were ignored altogether (PERT did assume stochastic activity durations, but used the expected value in a deterministic fashion). This simple model permitted solution as a network flow problem with an efficient polynomial-time algorithm.

A more realistic version of the project scheduling problem is the classical resource-constrained project scheduling problem, which takes into account the effects of nonavailability of sufficient resources [13]. This simple enhancement produces a drastic increase in computational complexity: the problem becomes hard and therefore classical optimal solution approaches such as mathematical programming formulations and enumerative methods quickly become infeasible for large problems and therefore heuristic techniques are employed. These heuristics usually take the form of simple dispatching rules (e.g., the popular MINSLK or minimum-slack rule) and do not guarantee optimality of solution [14], [54]. Morse and Whitehouse [45] describe the use of heuristic rule combinations for dispatching.

The classical resource-constrained project scheduling problem is generalized to deal with multiple resource types. [55] is a comparison of three enumeration-based optimization approaches to the multiple constrained-resource / single-project scheduling problem: bounded enumeration, branch and bound, and implicit enumeration. A further generalization of this problem permits resource utilization levels to vary during the execution of an activity instead of remaining constant [7]. An extreme case of this is preemption: the activity may be interrupted altogether and continued again at a later time.

Yet another version of the project scheduling problem requires the scheduling of multiple projects with individual project due-dates [44]. The dynamic project scheduling problem further assumes a stream of incoming projects governed by a probability distribution. In such a scenario, assigning a due-date to an incoming project is itself an important planning problem and has been tackled with the help of heuristic rules [15].

Several versions of the classical project scheduling problem result from scheduling objectives other than the most common one of minimizing overall project duration. A common objective is resource leveling: the problem of scheduling the activities so as to minimize resource loading peaks without increasing project duration beyond the original critical path duration [38]. "Crashing" of activities, i.e., the execution of critical activities at increased resource levels and extra cost in order to reduce finishing time is an integral part of the critical path method (CPM). Other scheduling objectives include maximizing resource utilization factors [37] and the net present value of the project [65]. In general, project scheduling is a multiobjective decision making problem with many of these objectives conflicting with each other.

Project scheduling is itself a part of the larger problem of project management, which is usually divided into four phases: planning, scheduling, chronicling, and analysis [67]. Project planning refers to the initial stage of the project during which the activities of the project are defined and their durations, precedence relationships, and other attributes are specified. In its full generality, this problem requires taking into account diverse features such as market conditions and the effects of hostile competition, and therefore it is considerably more unstructured than scheduling. During the chronicling and analysis stages project performance is monitored, extraordinary situations are detected, and deviations from schedule are corrected, by replanning and rescheduling if necessary. While initial planning and scheduling may take place over several months, it is obvious that replanning and rescheduling must be done in real-time to meet deadlines. Moreover, the need to disrupt the earlier schedule as little as possible becomes an important objective during rescheduling.

The range of project scheduling problems we have discussed in this section thus illustrates the entire spectrum of models 
from a simple deterministic world to a complex real world. Similar descriptions apply to other problem classes as well and therefore we provide only brief definitions of the job-shop scheduling and vehicle scheduling problems in the following sections.

\section{The Job-Shop Scheduling Class of Problems}

The classical general job-shop scheduling problem is defined as follows [20]. There arę $n$ jobs to be processed through $m$ machines. Each job must pass through each machine exactly once. The processing of a job on a machine is called an operation and requires a duration called the processing time. Technological constraints demand that each job should be processed through the machines in a specific order. Each job has a release time and a deadline. The general problem is to find a sequence in which jobs pass between the machines, which is compatible with the technological constraints and optimal with respect to some performance criterion. A variant of this problem, the flow-shop scheduling problem, arises if all jobs share the same processing order. In the open-shop problem, each job has to be processed on each machine, but there is no particular order to follow. Several other variants permit jobs to return to the same machines or to skip some machines, preemption of operations, schedule dependent processing times (due to setup and transport), inprocess inventories, and idle machines. The determination of the operation sequences, process routing, is the preliminary planning problem for job-shop scheduling.

Job-shop scheduling is a prototypical problem for a number of problems arising in several disciplines. Apart from other problems in production scheduling such as assembly line balancing and flexible manufacturing systems, job-shop scheduling has a close correspondence with project scheduling, time-tabling of lectures, and problems in computer science such as multiprocessor scheduling [12], [13], and this has fostered an exchange of scheduling strategies between these areas. Most of the classical optimization and heuristic methods described earlier for project scheduling have been applied to job-shop scheduling with similar results [3], [20], [26], [47]. [64] is a recent survey of production scheduling.

\section{Vehicle Routing and Scheduling}

Routing and scheduling problems [5] deal with a collection of entities (locations) requiring service by a fleet of vehicles. There may be precedence relationships requiring some entities to be serviced before others (e.g., picking up a consignment before delivering it), and service times for each entity. The problem is to provide a route and a schedule for each vehicle and its crew. A route specifies the sequence of locations to be visited, and the schedule identifies the times at which the activities at these locations are carried out.

A pure routing problem ignores temporal considerations, and hence is a pure planning problem by our classification. The most famous example of this class is the traveling salesman problem. Others are the Chinese Postman problem and the single/multiple depot node vehicle routing problems. A pure scheduling problem is one in which each serviceable entity has a prescribed (deterministic or randomly distributed) service time. Examples are mass transit crew/vehicle and air crew scheduling problems. Combined routing and scheduling problems include school bus scheduling, tractor-trailer routing and scheduling, etc. For a comprehensive description of optimal and graph theory based heuristic solution methods for vehicle and crew routing and scheduling problems, see [5].

\section{AI Approaches to PlanNing AND SCHEDUling PROBLEMS}

In this section we survey the main issues arising in the design of intelligent planning and scheduling systems. Table I lists some of the recent systems that have made important contributions to the development of planning and scheduling theory.

\section{A. Knowledge Representation}

Effective domain modeling and domain knowledge representation are central to the design of an intelligent planning/scheduling system. Several knowledge structures have been described in the literature: procedural nets, rules, frames/schema, scripts, and Petri nets. While analysis is easier with a uniform knowledge representation scheme, it is usually more appropriate to employ different types of knowledge structures to capture different types of domain information as it provides clarity of system organization, and consequently aids incremental program development and maintenance. The emphasis is on making knowledge explicit and on providing a natural reflection of the domain structure in the computer model. This gains the advantage of flexibility to do any kind of processing based on the representation: using sophisticated AI control procedures, generation of simulation scenarios, or plain old-fashioned optimization. A glance at Table II shows the diversity of the knowledge that may be required in a given problem. The OPAL [4] and SIPE [80] systems use multiple knowledge representation schemes. [63] provides an analysis of several schemes and their suitability for representing different categories of knowledge.

Perhaps the most popular representation scheme is the frame or schema-based approach which lends itself easily to an object-oriented style of programming. The popularity of this scheme may be attributed to the natural way in which it can model domain objects, both physical and abstract, e.g., machines, activities, events, etc., and its flexibility in handling the inheritance of properties and object specific procedures. MOLGEN [75], [76], ISIS [19], [72], Callisto [18], [67], and OPAL [4] all use schema-based representation.

Apart from domain objects, AI-based planning systems need to represent world states, the actions possible in each state, and the changes effected by each action (scheduling systems, however, usually start with a predefined activity graph and do not concern themselves with effects on world state). First-order predicate calculus is usually used to make assertions about the world; however, those assertions that relate to object attributes are best collected within an appropriate frame as mentioned in the preceding paragraph. The domain-independent planner SIPE [80] represents domain objects and their invariant prop- 
TABLE I

Some Al-Based Planning/Scheduling Systems

\begin{tabular}{|c|c|}
\hline Name & Features \\
\hline ISIS [19], [72] & $\begin{array}{l}\text { - knowledge-based factory scheduling } \\
\text { - robust representation of constraints } \\
\text { - hierarchical constraint-directed search }\end{array}$ \\
\hline OPIS [53], [72] & $\begin{array}{l}\text { —opportunistic scheduling } \\
\text { — reactive scheduling }\end{array}$ \\
\hline OPAL [4] & $\begin{array}{l}\text { - multiknowledge-based job-shop scheduling } \\
\text { - object-centered representation } \\
\text { - constraint-based analysis with heuristic } \\
\text { sequencing }\end{array}$ \\
\hline Battle [71] & $\begin{array}{l}\text { - expert system for resource allocation } \\
\text { - evidence functions for determining } \\
\text { effectiveness of an individual allocation } \\
\text { - informed search for finding composite } \\
\text { allocation }\end{array}$ \\
\hline NOAH, NONLIN [66] & $\begin{array}{l}\text { - network representation of world states } \\
\text { - concurrency in planning } \\
\text { - planning using macro actions }\end{array}$ \\
\hline MOLGEN [75], [76] & $\begin{array}{l}\text { - plans experiments in molecular genetics } \\
\text { - planning with constraints, meta-planning }\end{array}$ \\
\hline DEVISER [78] & - temporal planning \\
\hline SIPE $[80]$ & $\begin{array}{l}\text { - domain independent planner } \\
\text { - constraint language and interactivity }\end{array}$ \\
\hline ISA [33] & $\begin{array}{l}\text { - assists in scheduling computer assembly } \\
\text { - rule-based system }\end{array}$ \\
\hline
\end{tabular}

erties by nodes linked in a hierarchy, thus permitting it to retain the advantages of frame-based systems while retaining the power of predicate calculus for representing properties that vary. The classical "frame problem" arising in the context of representing actions is handled by using add-lists and deletelists, originally introduced in STRIPS [11], to represent the effects of actions.

Constraints form an important part of the knowledge base since the final acceptance of a schedule as satisfactory depends on how well the domain constraints have been met in the schedule. The complexity of planning and scheduling problems arises from the diversity of constraints generated by several levels of management: at the operational level, work-in-progress, machine and labor availability, technological constraints, etc., impose restrictions on the schedule, while at higher management levels, meeting due-dates, reducing peak resource loading levels, etc., impose further constraints on the schedule. In Table II we identify three types of constraints: necessities, preferences, and relaxations. Necessary constraints, e.g., technological constraints, must be satisfied by a schedule, whereas preferences are satisfied to the extent possible. Some constraints are soft: they may be relaxed under special circumstances. Capturing preference information may require the elicitation of a heuristic rule or the explicit specification of scheduling alternatives with a utility value for each alternative. The importance of constraints in obtaining a good schedule led to the development of the constraintbased systems MOLGEN and ISIS. Smith et al. [72] provide a detailed discussion of the nature of constraints arising in factory scheduling. SIPE also offers a domain-independent constraint language as one of its main contributions.

Representation and reasoning with imprecise and/or uncertain information is often facilitated by the use of fuzzy logic [81]. Table II lists several types of fuzzy statements. People
TABLE II

TYPES OF KNOWLedge IN AN INTELLIGENT SCHEDULING SySTEM

\begin{tabular}{|c|c|}
\hline Type & Examples \\
\hline \multicolumn{2}{|l|}{ Objects } \\
\hline classes & activity, network, resource \\
\hline instances & "act-3", "net-5", " "plumber-Joe" \\
\hline \multirow{2}{*}{\multicolumn{2}{|c|}{ Object attributes }} \\
\hline & \\
\hline facts & "The duration of act- 3 is 5 days" \\
\hline defaults & "Assume that duration of act- 3 is five days" \\
\hline factual rules & $\begin{array}{l}\text { "If the cement is of type } \mathrm{A} \text {, then act- } 3 \text { will } \\
\text { take } 5 \text { days" }\end{array}$ \\
\hline heuristic rules & $\begin{array}{l}\text { "If the cement is of type B, then assume } \\
\text { act- } 3 \text { takes } 5 \text { days" }\end{array}$ \\
\hline probabilistic information & "act-3 takes 5 days" with $C F=0.8$ " \\
\hline property inheritance & "design-activity" is a subclass "activity" \\
\hline definitions & A critical activity is one with zero slack. \\
\hline fuzzy facts & "Act-3 will take about 5 days" \\
\hline fuzzy rules & $\begin{array}{l}\text { "If the cement is of good quality then act- } 3 \\
\text { takes about } 3 \text { days" }\end{array}$ \\
\hline fuzzy probabilities & $\begin{array}{l}\text { "It is likely that act-3 will take about } \\
5 \text { days" }\end{array}$ \\
\hline \multicolumn{2}{|r|}{ J tays } \\
\hline $\begin{array}{l}\text { elementary structure } \\
\text { network structure }\end{array}$ & $\begin{array}{l}\text { a precedence link between two activities } \\
\text { the precedence network of a set of activities }\end{array}$ \\
\hline hierarchical structure & $\begin{array}{l}\text { the decomposition of an activity into a task } \\
\text { subnetwork }\end{array}$ \\
\hline group structure & $\begin{array}{l}\text { a set of tasks grouped according to } \\
\text { similarity of function }\end{array}$ \\
\hline linear orderings & $\begin{array}{l}\text { job prioritizations; resource listings } \\
\text { according to capacity. }\end{array}$ \\
\hline \multicolumn{2}{|l|}{ Procedural information } \\
\hline State information & \\
\hline past states & $\begin{array}{l}\text { "the previous activity was late due to a } \\
\text { labor strike" }\end{array}$ \\
\hline present states & a work-in-progress chart \\
\hline future states & $\begin{array}{l}\text { "more resources will be available next } \\
\text { week" }\end{array}$ \\
\hline Events & "start-act-3" \\
\hline Causal relations & Doing event- 2 results in state- 5 \\
\hline State evolution & $\begin{array}{l}\text { State }-5 \text { may lead to state }-9 \text { with probability } \\
0.7\end{array}$ \\
\hline Definitions & $\begin{array}{l}\text { Scheduling an activity means assigning a } \\
\text { feasible start time and a finish time to it. }\end{array}$ \\
\hline \multicolumn{2}{|r|}{ 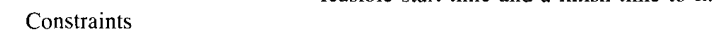 } \\
\hline necessities & act -3 must be finished by the 7 th \\
\hline preferences & If possible schedule job-7 before job-6 \\
\hline relaxations & $\begin{array}{l}\text { A few activities can miss their deadlines if } \\
\text { the overall delay is less than a week. }\end{array}$ \\
\hline \multicolumn{2}{|l|}{ Problem solving } \\
\hline \multicolumn{2}{|l|}{ knowledge } \\
\hline general search & A*, GBB algorithms $^{*}$ \\
\hline routine plans & $\begin{array}{l}\text { "The standard schedule for such a job } \\
\text { is ..." }\end{array}$ \\
\hline heuristic rules & the minimum-slack-first rule \\
\hline abnormality handling & $\begin{array}{l}\text { "In case of machine-4 breakdown, schedule } \\
\text { by procedure-3" }\end{array}$ \\
\hline conflict resolution & $\begin{array}{l}\text { "In general, meeting deadlines is more } \\
\text { important than having good resource } \\
\text { utilization" }\end{array}$ \\
\hline
\end{tabular}

can usually respond to questions more quickly and confidently in qualitative and imprecise terms, and this can result in both savings in time and ease of use. Hall [29] describes a prototype decision support system that uses fuzzy linguistic variables and production rules for strategic planning.

Some problem domains such as housing construction projects have a regular structure that does not vary much over individual problem instances, although specific parameters may change. This kind of knowledge may be captured using 
routine plans, i.e., a set of abstract, compiled, and partial plans containing information about specific components of the problem [9]. Routine plans can be used to encapsulate tested scheduling strategies that have evolved with experience and that do not require major changes. With the instantiation of the plan parameters, these "compiled" plans can be used to rapidly develop a complete schedule.

Most practical planning and scheduling systems contain a large body of heuristic knowledge, both about the domain and the problem solving techniques to be used. The necessity of making this heuristic information explicit cannot be overemphasized in view of the requirements of explanation capability and program maintenance. Rules are the most popular structure for representing heuristic information, though default heuristics can also be expressed using frames. Certainty factors may be associated with the rules. The intelligent scheduling assistant ISA [33], the parallel machine scheduling system PAMS [39], and the production scheduler of Bruno et al. [8] utilize a rule-based representation scheme.

Often, a combination of rules and frames is appropriate: frames provide a means of grouping related pieces of information, and representing the hierarchical/network structures that frequently arise in project management type problems (e.g., precedences, activity networks, descriptions at different levels of abstraction, etc.), whereas a logic-based representation aids the collection of other facts describing the problem, construction of a rulebase of heuristic scheduling polices, deduction, and question answering. Representing an activity duration with full richness requires the usage of either a crisp numerical value, a rule, a probability or a possibility distribution (for examples, see Table II), or in terms of an expression trading off resources used against duration ("15 man-hours"). These can be neatly integrated into the frame representation by treating the duration as a function which returns a value depending on the relevant information. Table II classifies the various types of knowledge that may appear in an intelligent scheduling system in a way that suggests a procedural, declarative, or object-oriented implementation.

The Petri net formalism [58] has gained prominence in recent years as a technique for the representation and analysis of concurrent processes. Petri nets are a graphical representation scheme well suited to hierarchical abstraction and as simulation input. Noronha and Sarma [50] look at Petri nets as a tool for hierarchical modeling and visual interaction in project management. A combination of Gantt chart and Petri net, the SSD graph, has been proposed as a tool for project scheduling and visualization [40].

\section{B. Hierarchical Representation and Search}

An important category of knowledge is that of the hierarchical structure of the domain. Hierarchical structure may occur in two ways: abstraction and problem decomposition. An abstraction hierarchy describes a problem at different levels of detail. During a search for information, the perceived complexity of a planning or scheduling problem depends on the resolution of the search, and using a wrong level of abstraction results either in uninformative data or in information overload. MOLGEN provides abstraction by means of the distinction between planning and meta-planning: planning is the creation and scheduling of actions in the domain, whereas meta-planning refers to knowledge about the problemsolving (planning) process itself [76]. However, Wilkins [80] argues that this distinction is not useful in domain independent planning.

Abstraction may also be obtained through the use of subplans implementing a given state change. For example, a subnetwork of activities in a project can be viewed as a single composite activity accomplishing all the results of the subactivities. The means-ends dimension is another line of hierarchical abstraction [61].

Hierarchical decomposition is distinct from hierarchical abstraction in that it refers to the divide-and-conquer strategy of AI, rather than to levels of detail [75]. Decomposition of a problem into nearly-independent subgoals can result in a substantial improvement in solution complexity [36]. Proper hierarchical abstraction of the domain may provide a clue to problem decomposition during search, e.g., as in the multilevel scheduling approach of ISIS [19]. ISIS also decomposes the scheduling problem by initially performing order prioritization. Similarly, OPIS [72] which is an extension of ISIS simplifies scheduling by identifying resource bottlenecks and assuming that these bottlenecks dominate the scheduling subproblems.

\section{Informed Search Methods}

Considerable research in $\mathrm{AI}$ has gone into the development of informed search strategies which utilize knowledge about the domain to speed up solution, instead of blindly exploring all possible alternatives. These algorithms have an important role to play when optimization of plans or schedules is required. Some of the well-known algorithms are the bestfirst search methods, $\mathrm{A}^{*}$ and $\mathrm{AO}^{*}$, and the game-tree search algorithms alpha-beta, B*, and SSS* [56]. The best-first search methods employ a heuristic evaluation function to select the most promising alternative at each decision point in the search process; the resource allocation system Battle [71] and the project scheduling algorithm described in [49] use this technique.

Search efficiency can be further improved by knowledgebased pruning of the search tree, i.e., by the elimination of several branches when it can be deduced that these options would not lead to an optimal solution. This approach is formalized by the generalized branch and bound algorithm (GBB) [46] which uses an "auxiliary database" to represent the domain knowledge that cuts down search. Both the $\mathrm{A}^{*}$ and the GBB algorithms produce optimal solutions with an appropriate choice of heuristic evaluation function, but a great virtue of these formulations is the ease with which they can be controlled to yield nonoptimal but good solutions at a reduction in computational cost. Beam search [52] is a nonoptimal search technique that retains only a fixed $k$ nodes (the beamwidth) at every level of the decision tree.

\section{Constraint Propagation and Analysis}

The importance of constraints in planning and scheduling problems (Section III-A) has resulted in considerable attention 
being focused on constraint-based search techniques. MOLGEN, ISIS, and OPAL are three systems with constraint analysis as their central feature. MOLGEN views planning as characterized by operations on constraints and presents "constraint posting" as an approach to hierarchical planning. Constraints are interpreted as a communication medium for expressing interactions between loosely coupled subproblems. Three operations on constraints are distinguished: 1) constraint formulation, which is the adding of new constraints as commitments in the design process; 2) constraint propagation, which is the creation of new constraints from old ones in a plan, and 3) constraint satisfaction, which is the operation of finding values for variables so that a set of constraints on the plan variables is satisfied. Since a constraint may be viewed as a partial description and commitment about objects and plan variables, constraint propagation is used to obtain as complete a description of the objects as possible; this is known as the least-commitment strategy, introduced in $\mathrm{NOAH}$ [66]. This process therefore narrows down the list of possible assignments at a decision point, and a commitment can then be made when it is more efficient to do so. Similarly, OPAL and DEVISER [78] propagate timing constraints and narrow down the window within which each activity may be scheduled.

Constraints not only place restrictions on the validity of a schedule, but can also play an active role in guiding schedule generation. ISIS views scheduling as a constraint-directed activity that is influenced by all relevant scheduling knowledge and uses the degree of satisfaction of resolved constraints to guide search.

\section{E. Reactive Planning, Real-Time Planning, Reaction Plans, Plan Caching, and Plan Compilation}

In recent years much attention has been focused on a newly proposed area of research known as reactive planning or "situated action" [1], [22], [53], [68], [69]. The general idea in this field is to build embodied agents that behave intelligently in physical surroundings [69]. Classical AI planners are criticized as consisting of a smart planning phase followed by a dumb execution phase which proves ineffective in real situations which are characteristically complex, uncertain, and immediate [1]. Agre and Chapman describe a system called Pengi that plays a video game possessing these three properties and express the view that a simple architecture consisting of a combinational network (called the central system) and peripheral systems (which processes early vision images) interacting with the world can participate in most forms of activity. Once again the blocks world problem appears as a testbed for this theory of activity and Chapman, describing a Pengi-like system Blockhead that solves this problem, states that what makes this problem easy for such systems is the absence of a representation or a world model and the use of vision instead. Similarly Schoppers makes a case for the use of reactive procedures and universal plans in the dynamic blocks world [68].

Ginsberg's criticism of universal plans [22] has lead to a debate on the practicality of the entire situated action enterprise [68], [22], [69], [23]. We believe that at the heart of this controversy lies confusion regarding the various terms in use, and the precise problem types whose solutions are under debate. For example, we find Ow et al. [53] referring to the Pengi system [1] as a reactive planner in which no advance planning is done, whereas the designers of this system are at pains to deny that Pengi is reactive, or indeed that its operation involves any plans at all [10]. Further, we find Schoppers drawing a distinction between reactive planning and reaction plans [69] but Ginsberg referring to the latter as pure reactive plans [23].

Clearly, the terminology requires settling down and we suggest distinguishing between the following terms: reactive plans and planning, reaction plans, real-time planning, plan compilation, and plan caching. Reactive planning is the process of replanning when an unexpected change in the environment during plan execution invalidates the old plan. The first point to keep in mind is that the entire issue of reactive planning arises due to uncertainty in the problem domain or nonspecification of the initial configuration, and therefore any criticism of classical (nonreactive) AI planning techniques on the grounds that they do not handle uncertainty and realtime requirements is moot, since classical $\mathrm{AI}$ techniques were designed for a different problem version altogether, viz., the blocks world problem in a known deterministic world discussed in Section II-A. It is not the techniques which are under question, but the modeling process: which model should be used in a given application (since simpler models may provide computational gains). Specialized solution techniques would have to be developed for each problem type on the known-deterministic world to hostile-real world spectrum, and, e.g., a criticism of a solution technique for the closed dynamic blocks world problem on the grounds that it does not think of solutions such as locking the baby or finding out who's baby it is to discipline it [23], is inappropriate.

Second, in what way is the reactive planning process different from nonreactive planning techniques? In some simple problem domains there is indeed no difference in the way one replans in reaction to an unexpected event and the way one does the initial planning. One such example is a variation of the classical traveling salesman problem in which the salesman suddenly finds during his (optimal) tour that his road is blocked by a landslide. In the resultant replanning problem, it is obvious that whatever solution techniques were used to develop the original tour can again be applied to solve the restated problem, since it is very similar to the original.

On the other hand, most real world problems have constraints peculiar to replanning. The most serious type of constraint is usually the time limit allowed for the selection of an appropriate response. Real-time planning and scheduling, in which the correctness of the solution depends on the timeliness of the action, has received considerable attention elsewhere in the computer science literature [74], but it appears that the situated action community in AI is independently rediscovering it under the umbrella of reactive planning.

What are the various techniques that may be employed to deal with real-time constraints during replanning? First, it may be possible to do the planning in advance, anticipating various events in the external world, and explicitly store the resulting strategy in an efficient manner. The latter step is called plan 
caching. Following our earlier definition of a strategy, the plan would be an action selection tree with each node representing the situation resulting from the previous action (incoming arc). The nodes at the topmost level are the various events that could occur in the environment, and each path in the tree represents an action sequence or "plan" in the narrower sense.

As a special case of this preplanning and caching technique, we might explicitly store only the first level of the strategy tree, which effectively is a table that matches each situation to a response action. The resultant system actually does no planning ("devising, designing, or formulating something to be done ..."): it is in essence a pattern classifier which transforms input situations (patterns) to output primitive actions (classes) and can be directly implemented with combinational circuits [68], [69]. These are called reaction plans, since they list the (predetermined) reaction appropriate to each situation. Note that reaction plans involve no replanning and hence are quite distinct from reactive planning systems which reactively plan, i.e., as a reaction to the event activate the planner and generate a new plan instead of just executing a primitive action according to a precomputed old plan.

This raises the issue of which parts of the (reaction) plan to precompute and cache, and when to reactively activate the planner. If the stored plan explicitly specifies a reaction for every possible world situation it is called a universal plan [68], [69]. Apart from the representational complexity this may entail, universal plans also inherit the defect that reaction plans may be myopic (since they specify only the immediate action to be taken, effectively looking at only the first level of the strategy tree). One way of handling representational complexity would be to trade off plan accuracy (selection of the best possible action in a given situation) in favor of a combinational circuit implementation with fewer gates; these are called approximate universal plans. Alternatively, the plan need not be made truly universal: in certain situations it might activate a planner instead of specifying the action to be executed. These plans are approximately universal plans. The practicability of automatically generating, storing, and using universal and approximately universal plans is what initially triggered off the controversial debate in this area and more research needs to be done before an answer becomes available.

Plan compilation is a generic term we use for a broad spectrum of techniques that embed problem solving or domain knowledge for planning into some low-level representation. Examples are the Pengi and Blockhead systems: although the authors claim that these systems do not perform any planning, there is a considerable amount of planning knowledge embedded in these systems. For example, Pengi's working is based on rules such as "when you are being chased, run away" [1] and hardwiring these rules with logic gates does not erase the fact that preplanning has been done and the resultant strategy embedded into the implementation. Similarly, Blockhead knows that to construct the goal stack it has to first find which block to copy, that it then has to find a similar block, then clear this block, and then place it on top of the partially completed goal stack, etc. [10]. Implementing the system using hardware and "visual routines processors" merely removes from sight that the higher level concepts involved are still the same: the most powerful planning system is one which encodes the maximum domain specific knowledge effectively. From this perspective we can also see that plan compilation may lead to inflexibility (because the basic plan algorithm is "hardwired," the system would have to be redesigned for a slightly different problem) although it yields a computational advantage. Other forms of plan compilation are the "recipes, directions, and instruction manuals" mentioned by Agre and Chapman [1], and the "routine plans" of Chandrasekaran [9].

In sum, problem solving (or just "activity") in dynamic real worlds often requires reactive planning. When these worlds are complex and immediate, effective reaction may require the use of plan caching, plan compilation, and real-time techniques. One often neglected constraint (which does not appear in toy problems such as the blocks world) in real-world replanning and rescheduling problems is the need to disrupt the previous plan or schedule as minimally as possible. For example, in a large project spanning several departments of an organization, there is a need to contain the effect of any changes to the original schedule, say within the section or workshop. This goal may even be preferred at the cost of other scheduling objectives. Ow et al. [53] and Smith [73] discuss the reactive scheduling methodology of the OPIS system which focuses on the resolution of constraint conflicts in the existing schedule.

\section{F. Opportunistic Planning and Scheduling}

While hierarchical planning is a "top-down" control structure, opportunistic planning [30] permits the shifting of focus among alternative plans. The system is organized as a number of independent modules known as "knowledge sources" or "specialists" using a central "blackboard" knowledge structure. The blackboard contains all the decisions generated during the planning process. Depending on the planning context, one of the decisions on the blackboard is kept in focus, and this decision triggers some of the plan specialists into generating tentative decisions for incorporation into the plan in progress. Decisions become final only after the planner has accepted an overall plan. Silverman et al. [70] look at distributed cooperative problem solving from a team-agent decision theoretic perspective and explain blackboard system generation with the project management metaphor.

A recent extension of ISIS, the Opportunistic Intelligent Scheduler (OPIS $\emptyset$ [72]) has been implemented with a blackboard style architecture. The knowledge sources implement alternative scheduling strategies that extend and revise a global set of scheduling hypotheses. Smith et al. report better performance than that of ISIS with this multiperspective scheduling approach. The domain-independent planner SIPE also provides a context mechanism that permits shifting of focus among alternatives; this cannot be done in systems that use a backtracking algorithm.

\section{G. Expert Systems and Decision Support Systems}

The rule-based expert systems architecture has proved successful in a number of applications including planning and scheduling. In this approach, domain data are represented as a collection of if-then rules, called the rulebase, and problem- 
solving (inferencing) is accomplished with a general purpose rule interpreter. These rules are activated by matching their pre- or postconditions with the given states or goals. Resolving conflicts among applicable rules is accomplished with the help of meta-rules. The explicitness of the knowledge, and the relative independence of the rules are the main advantages of this approach. However, rule-based systems turn out to be disadvantageous when complicated solution procedures or deep knowledge of the domain are required. ISA [33], PAMS [39], and [8] are three examples of rule-based scheduling systems. The expert systems approach has also been used for the selection of heuristic scheduling rules in a job-shop [2].

Closely related to expert systems are decision support systems (DSS) which are interactive computer-based systems that help decision makers confront ill-structured problems by providing direct access to data and analysis methods [60]. This is an active field of research and has evolved from early application-specific electronic data processing (EDP) systems through later management information system (MIS). Considerable attention has been focused in this area on understanding the semi-structured decision making process in order to provide a basis for decision aid. Intelligent decision support systems provide the unifying framework within which $\mathrm{Al}$ and expert systems technology can be synergistically integrated.

\section{H. Simulated Annealing, Neural Networks, and} Genetic Algorithms

Most scheduling optimization problems are NP-hard and local improvement procedures (hill-climbing) may lead to poor solutions. Recently, randomization-based procedures drawing analogies from statistical mechanics and genetics have been proposed as a means of overcoming local extrema and providing near-optimal solutions. The simulated annealing procedure is based on local improvement: given a solution (schedule), it tries to change the schedule slightly (say by exchanging the order of two parallel activities), accepting the change if the result is an improvement, but accepting the change with a small probability if the change is for the worse, in the hope that it may lead to a better solution later. The probability of accepting the new schedule is inversely related to the degradation in the quality of the schedule. A "cooling schedule" is used to provide controlled randomization of the search. Simulated annealing has been applied to production scheduling in an FMS [7], and in a neural network architecture based on the stochastic Hopfield model for job-shop scheduling [16]. Because of the large amount of fine grain parallelism and the resulting speed and robustness, neural networks have become an attractive technique for solving constraint satisfaction and optimization problems. Poliac et al. [59] describe the application of the parallel distributed processing approach to crew scheduling for restaurants. Their method uses three phases which are repeated until a satisfactory solution is obtained. The initial matching phase employs expert systems technology to match individuals to time shifts. This is followed by a search phase during which heuristics embedded in the network connections are used to generate a new schedule. In the assignment phase the new solution is evaluated and accepted or rejected based on an annealing scheme. Other neural network applications include robot path planning [32] and integer linear programming for job-shop scheduling [17]. Genetic algorithms [28] also use randomized selection and recombination of solutions to evolve a good solution. However, the practical usefulness of these optimization techniques still remains to be proved.

\section{Simulation}

A simulation module can provide valuable aid in a decision support system for planning and scheduling. Simulation provides support in three ways: 1) by generating scenarios that may result as the consequence of planning actions, known as "what-if" analysis; 2) as a means of handling uncertainty in the problem: e.g., uncertain activity durations are probabilistically sampled and used to generate a heuristic schedule in a Monte Carlo simulation to obtain a measure of the criticality of each activity: the frequency with which it appears on a critical path. Such information is useful in focusing attention on potential bottlenecks in the system and thereby in generating a more "robust" schedule. 3) In conjunction with an expert system module, simulation may be used to update the values of time-dependent variables in the expert system [51], or to make a particular decision requested by the module. For example, it may call for a simulation run to relatively prioritize contending actions based on environmental data, or to test the efficacy of several heuristics applicable in a given problem context. Expert systems can also act as an explanatory front-end to a complex simulator.

\section{SCheduling Issues in Project Management}

While considerable attention has been paid in the literature to AI applications in planning and production scheduling, the interest in the application of AI tools to project management is much more recent [18], [35], [49], [50]. Since projects are usually one-shot, project planning and scheduling are highly ill-structured problems and require a wider knowledge base and more powerful problem solving and structuring techniques than many other scheduling problems. In this section we broadly outline a strategy for the integration of AI techniques in a decision support system for project management.

The major components of an intelligent decision support system are the knowledge base, the problem solving control system, the user interface, and a simulation module. Problem solving for project management can be divided into two distinct parts: plan generation and scheduling. These two problem components are loosely coupled and may be implemented as two modules of the decision support system.

During plan generation, if the DSS is to permit the user to control and explore the problem situation, it has to provide assistance to the user in making first an abstract description of the problem, and then iteratively refining it. This suggests the need for a problem specification module within the problem solving system that interacts with the user via a natural language interface [62] or a constraint specification language. Work in this direction is exemplified by the goal-directed structuring system GODDESS [57] and the visual interactive planner VIPS [42]. Starting with the stated objectives, it 
focuses the user's attention on the most crucial issues and proceeds to unravel the more detailed means by which these objectives can be realized. Constraints and preferences are elicited, and contradictions are detected. The techniques used in the AI planning systems we have discussed, e.g., constraint propagation, are applied at this stage. While resource constraints are dealt with during the following scheduling stage (because their effects on timing are difficult to analyze), it is possible to analyze the effects of timing constraints such as deadlines during planning. For example, specifying a deadline on finishing an activity implies deadlines on finishing predecessor activities. Timing constraints on two activities may imply that one fully precedes the other. The result of this analysis leads to a plan specifying fully the precedence/timing restrictions on scheduling an activity. This plan can lead to a number of schedules, depending on other constraints (e.g., on resources) and on the scheduling objectives.

Another function of the problem solving control system is determining the solution strategy for the particular problem situation. It is aided in this function by a library of search algorithms, a user model, and appropriate control structures such as blackboards and agendas. The presence of multiple objectives and preferences in project management calls for considerable interaction with the user. Nondominated schedules must be presented from which the final schedule will be selected. If this set is too large, eliciting the decision maker's utility function is required, e.g., by Delphi methods [34]. The simulation module is used to deal with problem situations defying analytical solution and to generate "what if" scenarios for the decision maker. The simplicity and elegance of the Petri net formalism shows itself in this context as an intermediate representation between the problem solving system and the general purpose simulator [50].

\section{CONCLUDING REMARKS}

Scheduling is a rich area demanding the application of efficient methods to tackle the combinatorial explosion that results in real-world applications. Its richness also demands the use of sophisticated knowledge representation schemes. In spite of the diversity of scheduling problems we have tried to identify a common core of techniques from $\mathrm{AI}$ and expert systems that may be applied to ease the scheduler's burden. However, much remains to be done and future years should see the results of the application of ongoing active research in scheduling in the three areas of $\mathrm{AI}$, real-time systems, and decision support systems.

\section{REFERENCES}

[1] P.E. Agre and D. Chapman, "Pengi: An implementation of a theory of activity," in Proc. AAAI-87, 1987, pp. $268-272$.

[2] S. M. Alexander, "An expert system for the selection of scheduling rules in a job-shop," Comput. Ind. Eng., vol. 12, no. 3, pp. 167-171, 1987.

[3] R. Bellman, et al., Mathematical Aspects of Scheduling and Applications. Oxford, England: Pergamon, 1982.

[4] E. Bensana, G. Bel, and D. Dubois, "OPAL: A multi-knowledge-based system for industrial job-shop scheduling," Int. J. Production Res. vol. 26, no. 5, pp. $795-819,1988$.

[5] L. Bodin et al., "Routing and scheduling of vehicles and crews," Specia Issue of Comput. and Oper. Res., vol. 10, no. 2, 1983.
[6] D. A. Bourne and M.S. Fox, "Autonomous manufacturing: Automating the job-shop," IEEE Comput. Mag., pp. 76-86, Sept. 1984.

[7] P. Brandimarte et al., "FMS production scheduling by simulated annealing," in Proc. 3rd Int. Conf. Simulation in Manufacturing, 1987, pp. 235-245.

[8] G. Bruno, E. Antonio, and P. Laface, "A rule-based system to schedule production," IEEE Comput. Mag., vol. 19, pp. 32-39, July 1986.

[9] B. Chandrasekaran et al., "Building routine planning systems and explaining their behaviour," Int. J. Man-Machine Studies, vol. 30, no. 4 , pp. $377-398$, Apr. 1989.

[10] D. Chapman, "Penguins can make cake," AI Mag., vol. 10, no. 4, pp. 45-50, Winter 1989

[11] E. Charniak and D. McDermott, Introduction to Artificial Intelligence. Reading, MA: Addison-Wesley, 1985.

[12] E. G. Coffman, Computer and Job-shop Scheduling Theory. New York: Wiley, 1976.

[13] E. W. Davis, "Project scheduling under resource constraints_Fristorical review and categorization of procedures," AIIE Trans., vol. 5, no. 4, pp. 297-313, 1973.

[14] E.W. Davis and J.H. Patterson, "A comparison of heuristic and optimum solutions in resource-constrained project scheduling," Management Sci., vol. 21, no. 8, pp. 944-955, 1975.

[15] J. Dumond and V.A. Mabert, "Evaluating project scheduling and due date assignment procedures: An experimental analysis," Management Sci., vol. 34, no. 1, pp. 101-118, Jan. 1988.

[16] Y.S. Foo and Y. Takefuji, "Stochastic neural networks for solving job-shop scheduling, Part 1: Problem representation" and "Part 2: Architecture and simulations," in Proc. Int. Conf. Neural Networks, vol. 2, 1988 , pp. $275-290$.

[17] Y.S. Foo and Y. Takefuji, "Integer linear programming neural networks for job-shop scheduling," in Proc. Int. Conf. Neural Networks, vol. 2, 1988, pp. 341-348.

[18] M. S. Fox and J. McDermott, "The role of databases in knowledgebased systems," in On Knowledge Base Management Systems, M.L. Brodie and J. Mylopoulos, Eds. New York: Springer-Verlag, 1986, pp. $407-430$.

[19] M.S. Fox and S. F. Smith, "ISIS-A knowledge-based system for factory scheduling," Expert Syst., vol. 1, no. 1, pp. 25-49, July 1984.

[20] S. French, Sequencing and Scheduling: An Introduction to the Mathematics of the Job-shop. Chichester: Ellis Horwood, 1982.

[21] J.A. Gadsden, "Knowledge-based planning and replanning in naval command and control," in Proc. 4th Conf. AI Appl., 1988, pp. 286-292.

22] M. L. Ginsberg, "Universal planning: An (almost) universally bad idea," AI Mag., vol. 10, no. 4, pp. 40-44, 1989.

[23] __ "Ginsberg replies to Chapman and Schoppers," AI Mag., vol. 10, no. 4, pp. $61-62,1989$.

[24] F. Glover, "Future paths for integer programming and links to AI," Comput. and Oper. Res., vol. 13, no. 5, pp. 553-549, 1986.

[25] V. Goel and P. Pirolli, "Motivating the notion of generic design within information processing theory: The design problem space," $A I$ Mag., pp. 19-36, Spring 1989.

[26] R. L. Graham, et al., "Optimization and approximation in deterministic sequencing and scheduling: A survey," Ann. Discr. Math, vol. 5, pp. $287-326,1979$

[27] T. J. Grant, "Lessons for OR from AI: A scheduling case study," $J$. Operl. Res. Soc., vol. 37, pp. 41-57, 1986.

[28] J.J. Grefenstette, "Optimization of control parameters for genetic algorithms," IEEE Trans. Syst., Man, Cybern., vol. 16, no. 1, pp. 122-128, 1986.

[29] N. G. Hall, "Diagnosing problems with the user interface for a strategic planning fuzzy DSS," IEEE Trans. Syst., Man, Cybern., vol. 18, no. 4, pp. 638-646, Jul/Aug 1988.

[30] B. Hayes-Roth and F. Hayes-Roth, "Modelling planning as an incremental, opportunistic process," IJCAI, pp. 375-383, 1979.

[31] F. S. Hillier and G. J. Liebermann, Operations Research. San Francisco, CA: Holden-Day, 1974.

[32] C.C. Jorgenson, "Neural network representation of sensor graphs in autonomous robot path planning," in Proc. Int. Conf. Neural Networks, vol. 4,1987 , pp. $507-515$.

[33] J. J. Kanet and H. H. Adelsberger, "Expert systems in production scheduling," Euro. J. Opl. Res., vol. 29, pp. 51-59, 1987.

[34] Khorramshahgol et al., "An integrated approach to project evaluation and selection," IEEE Trans. Eng. Mgt., vol. 35, no. 4, Nov. 1988.

[35] S.O. Kim and M.J. Schniederjans, "Heuristic framework for the resource constrained multi-project scheduling system," Comput. and Oper. Res., vol. 16, no. 6, pp. 541-556, 1989.

[36] R.E. Korf, "Planning as search: A quantitative approach," Artif. Intell., vol. 33, pp. 65-88, 1987 
[37] I. Kurtulus and E.W. Davis, "Multi-project scheduling: Categorization of heuristic rules performance," Management $S_{c i}$, vol. 28, no. 2, pp. $161-171$, Feb. 1982.

[38] R.C. Leachman, "Multiple resource leveling in construction systems through variation of activity intensities," Naval Res. Logist. Quarterly, vol. 30 , pp. $187-198,1983$

[39] J.K. Lee and M.S. Suh, "PAMS: A domain-specific knowledgebased parallel machine scheduling system," Expert Syst., vol. 5, no. 3, pp. $198-214,1988$

[40] H. Lee-Kwang and J. Favrel, "The SSD graph: A tool for project scheduling and visualization," IEEE Trans. Eng. Mgt., vol. 35, no. 1 , pp. $25-30$, Feb. 1988.

[41] D. B. Lenat and E. A. Feigenbaum, "On the thresholds of knowledge," in Proc. IEEE Int. Workshop Artif. Intell. for Indust. Appl., 1988, pp. $291-300$.

[42] F. V. Martial and F. Victor, "An interactive planner for open systems," in Proc. Fourth Conf. Al Appl., IEEE, 1988, pp. 293-298.

[43] J. J. Moder and C. R. Phillips, Project Management with CPM and PERT. Reinhold, 1970.

[44] R.P. Mohanty and M.K. Siddiq, "Multiple projects-Multiple resources constrained project scheduling," Int. J. Production Res, vol. 27, no. 2 , pp. $281-292,1989$

[45] L. C. Morse and G. E. Whitehouse, "A study of combining heuristics for scheduling projects with limited resources," Comput. and Indust. Eng., vol. 15 , no. $1-4$, pp. $153-161,1988$

[46] D. S. Nau, V. Kumar, and L. Kanal, "General branch and bound, and its relation to $\mathrm{A}^{*}$ and $\mathrm{AO}^{*}$," Artif. Intell., vol. 23, pp. 29-58, 1984.

[47] Norbis and Smith, "Two level heuristic for the resource constrained scheduling problem," Int. J. Production Res., vol. 24, no. 5, pp. $1203-1219,1986$

[48] P. V. Norden, "On the integration of project management and information systems," in The Practical Application of Project Planning by Network Techniques. Halsted, 1972.

[49] S. J. Noronha and V. V.S. Sarma, "Artificial intelligence and knowledgebased approaches for scheduling problems in project management," in Proc. Int. Conf. Expert Syst. for Develop., Kathmandu, 1989, pp. $105-114$.

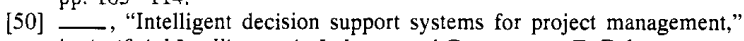
in Artificial Intelligence in Industry and Government, E. Balagurusamy, Ed. New Delhi, India: Macmillan, Nov. 1989.

[51] R. O'Keefe, "Simulation and expert systems-A taxonomy and some examples," Simulation, vol. 46, no. 1, pp. 10-16, Jan. 1986

[52] P.S. Ow and Morton, "Filtered beam search in scheduling," Int. J. Production Res., vol. 26, no. 1, Jan. 1988.

[53] P. S. Ow, S. F. Smith, and A. Thiriez, "Reactive plan revision," in Proc. AAAI-88, 1988, pp. 77-82.

[54] J.H. Patterson, "The effects of problem structure on heuristic performance," Naval Res. Logist. Quarterly, vol. 23, no. 1, 1976.

[55] _ "A comparison of exact approaches for solving the multiple constrained resource, project scheduling problem," Management Sci., vol. 30, no. 7, pp. 854-867, July 1984.

[56] J, Pearl, Heuristics, Reading, MA: Addison-Wesley, 1984.

[57] J. Pearl, A. Leal, and J. Saleh, "GODDESS: A goal-directed decision structuring system," IEEE Trans. Pattern Anal. Machine Intell., vol. 4, no. 3, pp. 250-262, May 1982.

[58] J. L. Peterson, Petri Net Theory and the Modeling of Systems. Englewood Cliffs, NJ: Prentice-Hall, 1981.'

[59] M. O. Poliac, E. B. Lee, J. R. Slagle, and M. Wick, "A crew scheduling problem," in Proc. Int. Conf. Neural Networks, vol. 4, 1987, pp. $779-786$.

[60] S. Raghavan and D. Chand, "A perspective on decision support systems," J. Comput. Soc. of India, vol. 18, no. 1, pp. 7-36, 1988.

[61] J. Rasmussen, "The role of hierarchical knowledge representation in decision making and system management," IEEE Trans. Syst., Man, Cybern., pp. 234-243, 1985.

[62] E. Rich, "Natural-language interfaces," IEEE Comput. Mag., vol. 17, pp. $39-47$, Sept. 1984

[63] G. A. Ringland and D. A. Duce, Eds., Approaches to Knowledge Representation, Research Studies Press Ltd., Wiley, New York, 1987.

[64] F. A. Rodammer and K. P. White, "A recent survey of production scheduling," IEEE Trans. Syst., Man, Cybern., vol. 18, no. 6, pp. 841-851, 1988.

[65] R. A. Russell, "A comparison of heuristics for scheduling projects with cash flows and resource restrictions," Management Sci., vol. 32, no. 10,

pp. $1291-1300$, Oct. 1986.
[66] E. Sacerdoti, A Structure for Plans and Behaviour. Amsterdam, The Netherlands: North-Holland, 1977.
[67] A. Sathi, M.S. Fox, and M. Greenberg, "Representation of activity knowledge for project management," IEEE Trans. Pattern Anal. Machine Intell., vol. 7, no. 5, pp. 531-552, 1985.

[68] M.J. Schoppers, "Universal plans for reactive robots in unpredictable environments," in Proc. Int. Joint Conf. Artif. Intell., 1987, pp. $1039-1046$.

[69] _, "In defence of reaction plans as caches," AI Mag., vol. 10, no. 4, pp. 51-60, Winter 1989

[70] B. G. Silverman, J.S. Chang, and L. Feggos, "Blackboard system generator (BSG): An alternative distributed problem solving paradigm," IEEE Trans. Syst., Man, Cybern., vol. 19, no. 2, pp. 334-355, Mar./Apr. 1989.

[71] J.R. Slagle and H. Hamburger, "An expert system for a resource allocation problem," Commun. ACM, vol. 28, no. 9, pp. 994-1004, Sept. 1985.

[72] S.F. Smith, M.S. Fox, and P.S. Ow, "Constructing and maintaining detailed production plans: Investigations into the development of knowledge-based factory scheduling systems," AI Mag., pp. 45-61, Fall 1986

[73] S. F. Smith, "A constraint-based framework for reactive management of factory schedules," in Intelligent Manufacturing, Proc. 1st Int. Conf. Expert Syst. and the Leading Edge in Production Planning, M. D. Oliff, Ed. Benjamin/Cummings, 1988, pp. 113-130.

[74] J.A. Stankovic and K. Ramamritham, Hard Real-Time Systems Tutorial, Computer Society Press of the IEEE, 1988.

[75] M. Stefik, "Planning with constraints," Artif. Intell., vol. 16, pp. 111 $140,1981$.

[76] _ "Planning and meta-planning," Artif. Intell., vol. 16, pp. $141-$ $170,1981$.

[77] F. B. Talbot, "Resource-constrained project scheduling: The nonpreemptive case," Management Sci., vol. 28, no. 10, pp. 1197-1210, Oct 1982.

[78] S.A. Vere, "Planning in time: Windows and durations for activities and goals," IEEE Trans. Pattern Anal. Machine Intell., vol. 5, no. 3 , pp. 246-267, May 1981.

[79] J.D. Wiest and F. K. Levy, A Management Guide to PERT/CPM. Englewood Cliffs, NJ: Prentice-Hall, 1969.

[80] D. E. Wilkins, "Domain-independent planning: Representation and plan generation," Artif. Intell., vol, 22, pp. 269-301, 1984.

[81] L. A. Zadeh, "Knowledge representation in fuzzy logic," IEEE Trans. Knowledge Data Eng., vol. 1, no. 1, pp. 89-100, Mar. 1989.

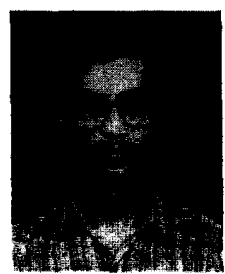

S.J. Noronha received the B.Tech. degree in electrical engineering from the Indian Institute of Technology, Madras, in 1987.

$\mathrm{He}$ is currently a doctoral candidate at the Indian Institute of Science, Bangalore. His research interests are in artificial intelligence, decision support systems, and operations research.

Mr. Noronha is a student member of the IEEE Computer Society.

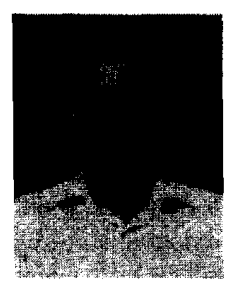

V.V.S. Sarma (SM'82) received the Ph.D. degree in electrical engineering in 1970 from the Indian Institute of Science (IISc), Bangalore.

He has been on the faculty at IISc since 1967 . He is presently a Professor in the Department of Com. puter Science and Automation. During 1984-1986, he was a Visiting Professor at the Centre for $\mathrm{Ad}$ vanced Computer Studies, University of Southwestern Louisiana, Lafayette. He has held summer appointments at Carnegie-Mellon and Duke Universities. His current interests are in artificial intelligence, expert systems, software engineering, and fault-tolerant computing. He is coauthor of a book on Reliability of Computer and Control Systems (NorthHolland, 1987)

Dr. Sarma is a Fellow of the Indian Academy of Sciences and the Indian National Science Academy, and a Senior Member of CSI. 